\title{
Saúde bucal de crianças e adolescentes hospitalizados: desafios e perspectivas
}

\author{
Oral health of hospitalized children and adolescents: \\ challenges and perspectives \\ Salud oral de los niños hospitalizados y adolescentes: desafíos y perspectivas \\ Niebla Bezerra de MELO ${ }^{1}$ \\ José de Alencar FERNANDES NETO ${ }^{1}$ \\ Jussara da Silva BARBOSA ${ }^{1}$ \\ Ítalo de Macedo BERNADINO ${ }^{1}$ \\ Thiago Santos de OLIVEIRA ${ }^{2}$ \\ Patrícia Meira BENTO ${ }^{3}$ \\ Francineide Guimarães CARNEIRO ${ }^{4}$ \\ ${ }^{I}$ Mestrando(a) do Programa de Pós-Graduação em Odontologia, Departamento de Odontologia, \\ Universidade Estadual da Paraíba, UEPB, 58429-500, Campina Grande - PB, Brasil \\ ${ }^{2}$ Cirurgião-Dentista pela Universidade Estadual da Paraíba, Departamento de Odontologia, \\ Universidade Estadual da Paraíba, UEPB, 58429-500, Campina Grande - PB, Brasil \\ ${ }^{3}$ Professora Doutora do Programa de Pós-Graduação em Odontologia, Universidade Estadual da Paraíba, UEPB, \\ 58429-500, Campina Grande - PB, Brasil \\ ${ }^{4}$ Professora Mestre do Curso de Odontologia, Departamento de Odontologia, Universidade Estadual da Paraíba, UEPB, \\ 58429-500, Campina Grande - PB, Brasil
}

\begin{abstract}
Resumo
Introdução: As condições de saúde bucal de crianças e adolescentes hospitalizados são preocupantes em virtude do aumento da suscetibilidade de problemas bucais devido ao período de internação. Objetivo: Avaliar a condição de saúde bucal de crianças e adolescentes hospitalizados. Material e método: Esta pesquisa tratou-se de um estudo do tipo transversal, composta por uma amostra de 51 crianças e adolescentes hospitalizadas. Foi avaliada as condições de saúde bucal dos pacientes através do Índice de Sangramento Gengival (ISG), Índice de Dentes Cariados, Perdidos e Obturados, na dentição decídua e permanente, (CPOD/ceo-d) e identificação de alterações estomatológicas. Os dados foram coletados através de uma entevista e avaliação clínica. Resultados: A média de idade foi de 7,39 anos. A maioria dos pacientes era do sexo feminino $(\mathrm{n}=23 ; 52,3 \%)$. Quase metade dos pacientes não realizava a higiene bucal no hospital $(\mathrm{n}=18 ; 40,9 \%)$. As médias de CPO-D, ceo-d e ISG foram, respectivamente, $2,77( \pm 3,47), 2,54( \pm 2,92)$ e $5,86( \pm 7,58)$. Verificou-se correlação positiva e significativa entre idade, CPO-D ( $\mathrm{r}=0,582 ; \mathrm{p}<0,01)$ e ISG $(\mathrm{r}=0,552 ; \mathrm{p}<0,01)$. Conclusão: Foi constatada a deficiência das condições de saúde oral das crianças e adolescentes hospitalizados, enfatizando dessa forma os cuidados com a saúde bucal no ambiente hospitalar.

Descritores: Criança Hospitalizada; Adolescente Hospitalizado; Saúde Bucal; Odontologia Preventiva; Assistência Odontológica.
\end{abstract}

\begin{abstract}
Introduction: The oral health conditions of hospitalized children and adolescents are worrisome due to the increase in the susceptibility of oral problems due to the period of hospitalization. Objective: To evaluate the oral health status of hospitalized children and adolescents. Material and methods: This study was a cross-sectional study, comprising a sample of 51 hospitalized children and adolescents. The oral health conditions of the patients were evaluated through the Gingival Bleeding Index (ISG), Caries, Lost and Sealed Index of the deciduous and permanent dentition (CPOD / ceo-d) and identification of stomatological alterations. Data were collected through an interview and clinical evaluation. Results: The mean age was 7.39 years. The majority of the patients were female $(\mathrm{n}=23 ; 52.3 \%)$. Almost half of the patients did not perform oral hygiene in the hospital ( $\mathrm{n}$ $=18,40.9 \%)$. The mean CPO-D, ceo-d and ISG were, respectively, $2.77( \pm 3.47), 2.54( \pm 2.92)$ and 5.86 $( \pm 7.58)$. There was a positive and significant correlation between age, CPO-D $(r=0.582, \mathrm{p}<0.01)$ and ISG $(\mathrm{r}=0.552, \mathrm{p}<0.01)$. Conclusion: It was verified the deficiency of the oral health conditions of hospitalized children and adolescents, emphasizing in this way oral health care in the hospital environment.
\end{abstract}

Descriptors: Child, Hospitalized; Adolescent, Hospitalized; Oral Health; Preventive Dentistry; Dental Care.

\section{Resumen}

Introducción: El estado de salud bucal de los niños y adolescentes hospitalizados son motivo de preocupación debido al aumento de la susceptibilidad a problemas dentales debido a la hospitalización. Objetivo: Evaluar el estado de salud bucal de los niños y adolescentes hospitalizados. Material y método: Esta investigación aborda es un estudio transversal, que consiste en una muestra de 51 niños y adolescentes hospitalizados. Se evaluó el estado de salud bucal de los pacientes a través del índice de sangrado gingival (GBI), Dientes Índice de dientes cariados, perdidos y llenos en la dentición decidua y permanente (CPO-D / c-d) y cambios estomatológicos identificación. Los datos fueron recolectados a través de una entevista y evaluación clínica. Resultados: La edad media fue de 7,39 años. La mayoría de los pacientes eran mujeres $(\mathrm{n}=23 ; 52,3 \%)$. Casi la mitad de los pacientes no se realizó la higiene oral en el hospital $(\mathrm{n}=18 ; 40,9 \%)$. El CPOD promedio, CPOD y el ISG fueron, respectivamente, $2,77( \pm 3,47), 2,54( \pm 2,92)$ y $5,86( \pm 7,58)$. Hubo una correlación positiva y significativa entre la edad, CPO-D $(\mathrm{r}=0,582 ; \mathrm{p}<0,01)$ y el ISG $(\mathrm{r}=0,552 ; \mathrm{p}<0,01)$. Conclusión: la carencia de condiciones de salud oral de los niños y adolescentes hospitalizados se ha encontrado, enfatizando así el cuidado de la salud oral en el hospital.

Descriptores: Niño Hospitalizado; Adolescente Hospitalizado; Salud Bucal; Odontología Preventiva; Atención Odontológica.

\section{INTRODUÇÃO}

A saúde bucal está entre os aspectos fisiológicos de grande importância para o crescimento e desenvolvimento das crianças e adolescentes ${ }^{1,2}$. Efeitos de alterações na saúde bucal não estão limitados apenas à boca, podendo levar a quadros infecciosos, resultando em comprometimento sistêmico ${ }^{3,4}$.
Pacientes hospitalizados estão sujeitos a uma série de fatores que contribuem negativamente para a sua saúde bucal, além de estarem mais susceptíveis à infecção ${ }^{3,5}$.

Mudanças extremas nos horários das refeições e nos hábitos alimentares, introdução de medicamentos na rotina diária, o estresse pela hospitalização, a indisposição 
ocasionada pela doença e a estadia em um ambiente diferente do habitual levam a uma subvalorização dos cuidados bucais dos pacientes internados em ambiente hospitalar ${ }^{1,6,7}$.

O acúmulo de placa e higiene bucal deficiente são os problemas mais evidentes encontrados durante avaliação odontológica de pacientes hospitalizados ${ }^{3,8,9}$. Dessa forma é de suma importância a realização de procedimentos de promoção de saúde bucal como profilaxia dentária, técnicas de escovação e aplicação tópica de flúor ${ }^{10,11,12}$.

A odontologia hospitalar tem como objetivo atuar nas alterações de saúde bucal de pacientes hospitalizados através de uma equipe multidisciplinar, garantindo uma abordagem integral do indivíduo ${ }^{13,14,15}$

O tratamento odontológico nesses pacientes, assim como, ações de promoção de saúde, contribuem para a prevenção e/ou melhora da condição sistêmica, diminuindo a incidência de infecções, necessidade de antibióticos sistêmicos e sua conseguinte mortalidade, resultando benefícios significativos ${ }^{16,17,18}$

No entanto, percebe-se que Odontologia ainda tem muitos obstáculos a vencer no contexto hospitalar ${ }^{11,19}$. Estudos evidenciam pouco investimento em recursos e treinamento dos profissionais nas instituições hospitalares, para esse contexto ${ }^{11,14,15}$.

Diante disso, esse estudo teve como objetivo avaliar as condições de saúde bucal de crianças e adolescentes hospitalizados, investigando possíveis correlações entre variáveis sociodemográficas e os hábitos de higiene oral dos mesmos. A fim de dá subsídios para o planejamento e desenvolvimento de futuras ações voltadas à odontologia no ambiente hospitalar.

\section{MATERIAL E MÉTODO}

Esta pesquisa tratou-se de um estudo do tipo transversal, realizada no Hospital Municipal da Criança e do Adolescente - Dr. Severino Bezerra de Carvalho no munícipio de Campina Grande, Paraíba. A população do estudo compreendeu 51 crianças e adolescentes internadas no hospital durante o período de janeiro a maio de 2015 .

De acordo com a resolução do Conselho Nacional de Saúde (CNS) 466/12, esta pesquisa foi cadastrada na Plataforma Brasil e aprovada pelo Comitê de Ética em Pesquisa da Universidade Estadual da Paraíba tendo como protocolo o número: 37237214.2.0000.5187.

A coleta de dados foi realizada por dois pesquisadores treinados, através de um formulário pré-elaborado, onde foram colhidas informações sociodemográficas, hábitos de higiene oral e saúde geral do paciente. Os prontuários médicos de cada paciente foram consultados, sempre que necessário. Além disso, foram realizados exames intrabucais, a fim de se verificar as condições de saúde bucal por meio da identificação de lesões de tecido mole, Índice de Dentes Cariados, Perdidos e Obturados (CPOD/ceo-d), e Índice de Sangramento Gengiva (ISG).

Foi realizado previamente um estudo piloto com 10 pacientes internos, a fim de garantir a calibração dos pesquisadores. Os dados do estudo piloto não foram incluídos nos resultados finais da pesquisa.

A metodologia utilizada para a realização dos exames intrabucais foi a preconizada pela Organização Mundial da Saúde (OMS) e pelo Manual do Examinador SBBRASIL $2012^{20}$. Os exames foram realizados utilizando-se espelho bucal e sonda da OMS, sob a luz natural, com o examinador devidamente paramentado, de pé e o paciente sentado. Os diferentes espaços dentários foram abordados, sistematicamente, a fim de se verificar presença de cárie (com cavitação evidente), restauração, dentes perdidos, incluindo neste grupo os elementos com necrose pulpar, com coroa destruída, os com extração indicada. As possíveis lesões de mucosa foram verificadas através do exame detalhado das mucosas bucais e estruturas anatômicas.

$O$ Índice de sangramento gengival foi avaliado por meio da sonda OMS, inserida levemente na entrada do sulco gengival e percorrida por toda a extensão do dente, seguindo a configuração anatômica da superfície da gengiva. Após esse procedimento, aguardava-se cerca de 10 a 30 segundos para a análise de presença ou ausência de sangramento da gengiva marginal ${ }^{21}$.

Os dados foram tabulados com o auxílio do software Microsoft Excel 2016 e importados para análise estatística utilizando software IBM SPSS Statistics versão 20.0 (IBM Corp., Armonk, NY, USA). Inicialmente, realizou-se a análise estatística descritiva objetivando caracterizar a amostra. Foram calculadas as frequências absolutas e percentuais para as variáveis qualitativas, bem como as medidas de tendência central (média, mediana) e de variabilidade (desvio padrão) para as variáveis quantitativas. A normalidade dos dados não foi verificada após realizar o teste de Shapiro-Wilk. As variáveis idade, tempo de internação, CPO-D, ceo-d e ISG em sua forma não categorizada foram analisadas por meio do teste de correlação de Spearman (dados não paramétricos) ${ }^{22}$.

\section{RESULTADOS}

A média de idade foi de 7,39 anos, variando de 2 a 17 anos. A maioria dos pacientes era do sexo feminino $(\mathrm{n}=23$; $52,3 \%)$, estava acompanhada da mãe $(\mathrm{n}=30 ; 68,2 \%)$ e foram diagnosticados com doenças respiratórias $(n=21$; 47,7\%). A Tabela 1 mostra a distribuição das crianças e dos adolescentes de acordo com as características sociodemográficas, tempo de internação doença diagnosticada.

Tabela 1. Distribuição das crianças e dos adolescentes de acordo com as características sociodemográficas, tempo de internação e doença diagnosticada

\begin{tabular}{lccc}
\hline \multicolumn{1}{c}{ Variáveis } & Média & Mediana & Desvio Padrão \\
\hline Idade & 7,39 & 6,50 & $\pm 3,83$ \\
Tempo de internação atual (dias) & 3,16 & 2,00 & $\pm 2,05$ \\
\hline & N & $\%$ \\
\hline Sexo & & \\
Masculino & 21 & 47,7 \\
Feminino & 23 & 52,3 \\
Moradia (procedência) & & \\
Zona urbana & 32 & 72,7 \\
Zona rural & 12 & 27,3 \\
Grau de parentesco do acompanhante & & \\
Mãe & 30 & 68,2 \\
Avós & 6 & 13,6 \\
Irmãos & 2 & 4,5 \\
Outro (primo, tio, noivo) & 4 & 9,1 \\
Nenhum grau & 2 & 4,5 \\
Escolaridade do acompanhante & & \\
Ensino fundamental incompleto & 15 & 34,1 \\
Ensino fundamental completo & 6 & 13,6 \\
Ensino médio incompleto & 6 & 13,6 \\
Ensino médio completo & 16 & 36,4 \\
Nenhuma escolaridade & 1 & 2,3 \\
Doença diagnosticada & & \\
Doenças respiratórias & 21 & 47,7 \\
Infecções do trato urinário & 6 & 13,6 \\
Enteroinfecções/Desidratação & 3 & 6,8 \\
Afeç̧ões cutâneas & 3 & 6,8 \\
Sem diagnóstico & 11 & 25,0 \\
\hline
\end{tabular}

A maior parte dos pacientes relatou utilizar apenas a escova e creme dental durante a higienização dentária $(\mathrm{n}=43 ; 97,7 \%)$ e apenas $1(2,3 \%)$ paciente relatou ter 
recebido orientação de higiene bucal no hospital. A Tabela 2 apresenta a distribuição das crianças e dos adolescentes de acordo com as práticas de higiene bucal. Um total de 32 $(72,7 \%)$ dos pacientes possuía algum tipo de alteração em tecido mole, sendo língua saburrosa $(n=20 ; 45,5 \%)$ a mais frequente. As médias de CPO-D, ceo-d e ISG foram, respectivamente, $2,77( \pm 3,47), 2,54( \pm 2,92)$ e $5,86( \pm 7,58)$ (Tabela 3). Verificou-se correlação positiva e significativa entre idade, CPO-D $(r=0,582 ; \mathrm{p}<0,01)$ e ISG $(r=0,552 ; \mathrm{p}$ $<0,01)$ (Tabela 4).

Tabela 2. Distribuição das crianças e dos adolescentes de acordo com hábitos de higiene bucal.

\begin{tabular}{|c|c|c|}
\hline Variáveis & $\mathbf{N}$ & $\%$ \\
\hline \multicolumn{3}{|l|}{ Visita anterior ao dentista } \\
\hline Sim & 29 & 65,9 \\
\hline Não & 15 & 34,1 \\
\hline \multicolumn{3}{|l|}{ Frequência de escovação diária } \\
\hline $1 \mathrm{vez}$ & 10 & 22,7 \\
\hline 2 vezes & 15 & 34,1 \\
\hline 3 vezes & 13 & 29,5 \\
\hline Mais de 3 vezes & 5 & 11,4 \\
\hline Nenhuma & 1 & 2,3 \\
\hline \multicolumn{3}{|c|}{ Quem executa a escovação do paciente? } \\
\hline Pais/responsável & 31 & 70,5 \\
\hline Próprio paciente & 12 & 27,3 \\
\hline Ninguém & 1 & 2,3 \\
\hline \multicolumn{3}{|c|}{ Instrumentos para higienização dentária* } \\
\hline Escova dental & 43 & 97,7 \\
\hline Creme dental & 43 & 97,7 \\
\hline Colutório bucal & 5 & 11,4 \\
\hline Fio dental & 7 & 15,9 \\
\hline \multicolumn{3}{|l|}{ Realiza higiene bucal noturna? } \\
\hline Sim & 31 & 70,5 \\
\hline Não & 13 & 29,5 \\
\hline \multicolumn{3}{|c|}{ Trouxe escova dental para o hospital? } \\
\hline Sim & 28 & 63,6 \\
\hline Não & 16 & 36,4 \\
\hline \multicolumn{3}{|l|}{ Realiza higiene bucal no hospital? } \\
\hline Sim & 26 & 59,1 \\
\hline Não & 18 & 40,9 \\
\hline \multicolumn{3}{|c|}{ Recebeu orientação de higiene bucal no hospital? } \\
\hline Sim & 1 & 2,3 \\
\hline Não & 43 & 97,7 \\
\hline
\end{tabular}

Tabela 3. Distribuição das crianças e dos adolescentes de acordo com a presença de alterações bucais, CPO-D, ceo-d e ISG.

\begin{tabular}{|c|c|c|c|}
\hline \multicolumn{2}{|c|}{ Variáveis } & $\mathbf{N}$ & $\%$ \\
\hline \multicolumn{4}{|c|}{ Alteração em tecido mole } \\
\hline \multicolumn{2}{|l|}{ Sim } & 32 & 72,7 \\
\hline \multicolumn{2}{|c|}{ Não } & 12 & 27,3 \\
\hline \multicolumn{4}{|c|}{ Língua saburrosa } \\
\hline \multicolumn{2}{|l|}{ Sim } & 20 & 45,5 \\
\hline \multirow{2}{*}{\multicolumn{2}{|c|}{$\begin{array}{l}\text { Não } \\
\text { Lábios ressecados }\end{array}$}} & 24 & 54,5 \\
\hline & \multicolumn{3}{|c|}{ Lábios ressecados } \\
\hline \multicolumn{2}{|l|}{ Sim } & 11 & 25,0 \\
\hline \multirow{2}{*}{\multicolumn{2}{|c|}{$\begin{array}{l}\text { Não } \\
\text { Línqua fissurada }\end{array}$}} & 33 & 75,0 \\
\hline & & & \\
\hline \multicolumn{2}{|l|}{ Sim } & 2 & 4,5 \\
\hline \multirow{2}{*}{\multicolumn{2}{|c|}{$\begin{array}{l}\text { Não } \\
\text { Língua geográfica }\end{array}$}} & 42 & 95,5 \\
\hline & & & \\
\hline \multicolumn{2}{|l|}{ Sim } & 3 & 6,8 \\
\hline \multirow[t]{2}{*}{ Não } & & 41 & 93,2 \\
\hline & Média & Mediana & $\begin{array}{l}\text { Desvio } \\
\text { padrão }\end{array}$ \\
\hline CPO-D & 2,77 & 2,00 & $\pm 3,47$ \\
\hline ceo-d & 2,54 & 2,00 & $\pm 2,92$ \\
\hline ISG & 5,86 & 2,50 & $\pm 7,58$ \\
\hline
\end{tabular}

Tabela 4. Correlação de Spearman entre idade, tempo de internação, CPO-D, ceo-d e ISG.

\begin{tabular}{lccccc}
\hline \multicolumn{1}{c}{ Variáveis } & Idade & $\begin{array}{c}\text { Tempo de } \\
\text { internação }\end{array}$ & CPO-D & ceo-d & ISG (\%) \\
\hline Idade & 1,000 & - & - & - & - \\
Tempo de Internação & $-0,326^{*}$ & 1,000 & - & - & - \\
CPO-D & $0,582^{* *}$ & $-0,016$ & 1,000 & - & - \\
ceo-d & 0,123 & 0,023 & $0,612^{* *}$ & 1,000 & - \\
ISG (\%) & $0,552^{* *}$ & $-0,289$ & 0,152 & 0,228 & 1,000 \\
\hline
\end{tabular}

Nota. *Correlação significativa ao nível de $\mathrm{p}<0,05$

**Correlação significativa ao nível de $\mathrm{p}<0,01$

\section{DISCUSSÃO}

A importância da higiene bucal para garantir o bemestar, a prevenção de doenças sistêmicas e a melhora na recuperação do paciente hospitalizado não é algo ainda consolidado no Brasil ${ }^{2}$.

A implantação da Odontologia se faz necessária no contexto hospitalar e sua consolidação implica em melhorias de infraestrutura e investimento intelectual ${ }^{18}$.

Os resultados deste estudo corroboram com às características da população que habitualmente busca atendimento hospitalar pelo Sistema Único de Saúde (SUS), a maioria de baixa renda e de baixa escolaridade, e de acordo com levantamentos epidemiológicos, aquela em que há maior prevalência de doenças bucais ${ }^{11}$.

Observa-se que grande parte dos pacientes avaliados neste estudo veio da zona urbana (Tabela 1). Este fato pode ser explicado pelas dificuldades que a população rural ainda enfrenta no acesso aos serviços de saúde ${ }^{16,23}$.

Sobre a questão de hábitos de higiene bucal, a maior parte dos pacientes avaliados afirmou escovar os dentes 2 vezes ao dia (Tabela 2). No entanto, em relação à escovação dentária durante $\mathrm{o}$ período de internação hospitalar percebeu-se que 40,9\% dos internos não realizavam o procedimento (Tabela 2), fato semelhante ao detectado pelo estudo de Silveira et al. ${ }^{4}$. Além do mais quanto à utilização do fio dental a pesquisa evidencia uma baixa adesão a essa prática (Tabela 2).

A literatura aponta a correlação entre complicações decorrentes da falta de higiene bucal e o aumento da permanência hospitalar em 6,8 a 30 dias, e que o controle mecânico da placa bacteriana, por meio da escovação e uso de fio dental, associado ao uso de substâncias químicas (clorexidina $0,12 \%$ ) é fundamental $^{24,25}$.

Ainda analisando os hábitos de higiene bucal (Tabela 2), verifica-se que praticamente toda a amostra não recebeu nenhuma orientação sobre cuidados com a saúde oral, durante o período de hospitalização, corroborando com os estudos de Ximenes et al. ${ }^{26}$ e Rodrigues et al. ${ }^{8}$. No entanto o resultado difere completamente dos achados de Silveira et al. $^{4}$ onde $100 \%$ da amostra estudada afirmou receber orientações de saúde bucal. Isso se deve ao fato do hospital em questão, avaliado por Silveira et al. ${ }^{4}$, ter a presença do cirurgião-dentista no quadro de funcionários, diferentemente do local desta pesquisa, reforçando a importância deste profissional na equipe hospitalar.

No ambiente hospitalar, o paciente internado deve ser monitorado e os cirurgiões-dentistas têm o papel fundamental na avaliação da saúde oral, tendo em vista que estas avaliações são essenciais para os cuidados da saúde geral e no atendimento do paciente como um todo, pois diversas manifestações na cavidade oral podem surgir a partir das condições sistêmicas ${ }^{1,10,15}$.

As manifestações bucais podem ser resultantes do comprometimento do sistema imunológico, podendo ser causadas por bactérias, fungos e vírus, ou serem de natureza neoplásica ${ }^{27}$. Tradicionalmente, a mucosa da cavidade bucal tem sido encarada como um reflexo do estado geral de saúde $^{28}$. Numerosas alterações da mucosa bucal podem ser encontradas em crianças, desse modo, profissionais da saúde devem ser capazes de detectá-las para o estabelecimento do diagnóstico correto e o tratamento adequado ${ }^{29}$.

Examinando as possíveis alterações de tecido mole nos pacientes deste estudo verificou-se a presença de algum tipo de lesão em $72,7 \%$ da população (Tabela 3), aproximando-se do valor encontrado no estudo realizado por Cruz et al. ${ }^{9}$.

Dentre as alterações observadas em tecido mole, a mais comum foi a língua saburrosa, fato que difere ao encontrado por Yilmaz et al. ${ }^{30}$, Marjorana et al. ${ }^{31}$, Bessa et 
al. ${ }^{32}$ e Crivelli et al. ${ }^{33}$ que registraram lesões mais frequentes a candidíase oral, língua geográfica e lesões de origem infeciosa, respectivamente.

A língua saburrosa é formada basicamente por restos alimentares, células descamadas, fungos, bactérias e enzimas ativas que participam do processo da digestão. Estudos relatam a importância da realização da limpeza da língua para a remoção dessa saburra, também denominada biofilme lingual. Reforçando a orientação de higiene bucal no ambiente hospitalar ${ }^{34}$.

A higiene bucal deficiente e o acúmulo de placa são os problemas mais evidentes encontrados durante o exame odontológico de pacientes hospitalizados ${ }^{8}$. De acordo com Panagakos e Scannapieco ${ }^{35}$ o acúmulo de placa dental leva à inflamação periodontal, sendo a gengivite a manifestação inicial deste processo. Justificando-se assim a alta média de sangramento gengival nos pacientes avaliados (Tabela 3 ).

Os dados da pesquisa relataram uma tendência de que quanto maior a idade dos pacientes, maiores são os valores de CPO-D e ISG, consequentemente, pior é a condição de saúde bucal (Tabela 4).

Sabe-se que a inflamação dos tecidos gengivais pode ser encontrada em todas as idades, desde que a placa bacteriana se acumule por certo período de tempo nos dentes $^{36}$. No entanto, dados epidemiológicos revelam que problemas periodontais aumentam com a idade ${ }^{20}$, fato evidenciado nesta pesquisa.

A cárie dentária, principal agravo em saúde bucal da infância, representa um processo patológico passível de prevenção, sendo fundamental o controle da microbiota envolvida em sua etiologia, através da higiene bucal e controle de placa ${ }^{37}$.

O cirurgião dentista exerce importante função na equipe de saúde, para garantir a atenção integral aos indivíduos, minimizando desconfortos ocasionados por problemas de ordem geral e bucal ${ }^{10,11,38}$. Contudo, muitas vezes há um negligencia mento da participação do profissional da Odontologia frente à atenção hospitalar ${ }^{14}$.

Desta forma deve-se estimular a formação de equipes multidisciplinares no ambiente hospitalar no intuito de enfatizar a adoção de medidas de promoção de saúde, incluindo a saúde bucal, na tentativa de auxiliar na melhoria do quadro sistêmico durante a hospitalização, sendo essencial incluir todos os sujeitos envolvidos: criança, cuidador e profissionais da saúde ${ }^{8}$.

Alguns pacientes não aceitaram a realização do exame, ocasionando em perdas na amostra. Além disso, devido à recorrência de algumas lesões de mucosa bucal, sua verdadeira ocorrência pode ter sido declinada neste estudo, uma vez que lesões ativas poderiam não estar presentes no momento do exame.

\section{CONCLUSÃO}

Foi constatada a deficiência das condições de saúde oral das crianças e adolescentes avaliados, enfatizando dessa forma a necessidade da presença de um cirurgião-dentista e de um protocolo de higiene bucal no ambiente hospitalar para melhores condições de saúde bucal das pacientes internados.

\section{REFERÊNCIAS}

1. Aranega AM, Bassi APF, Ponzoni D, Wayama MT, Esteves JC, Junior IRG. Qual a importância da Odontologia Hospitalar? Rev Bras Odontol. 2012; 69(1):90-3.
2. Lima MCPS, Lobo INR, Leite KVM, Muniz GRL, Steinhauser HC, Maia PRM. Condição de saúde bucal de crianças internadas no Hospital Municipal Infantil de Imperatriz - Maranhão. Rev Bras Odontol. 2016; 73(1):24-9.

3. Amaral KC, Tenório MDH, Dantas AB. Condição de saúde bucal de crianças internas em hospitais da cidade de Maceió-AL. Odontol Clín-Científ. 2006; 5(4):267-73.

4. Silveira ER, Costa FS, Azevedo MS, Schardosin LR. Perfil de saúde bucal de crianças internadas em Unidade de Pediatria de um Hospital Escola. Pediatr Mod. 2014; 50(12):546-52.

5. Lamha ASF, Costa FOC. Utilização do "Brinquedo" como recurso mediador na promoção de saúde bucal em pacientes internados na enfermaria pediátrica do HU/UFSC. R Bras Ci Saúde. 2012; 16(3):285-94.

6. Arcênio RA, Oliveira MF, Villa TCS. Internações por tuberculose pulmonar no estado de São Paulo no ano de 2004. Cien Saúde Coletiva. 2007; 12(2):409-17.

7. Silva MJCN, Costa CPS, Sá FAO, Borges LO, Sauáia TS. Por que devemos nos preocupar com a saúde bucal de crianças hospitalizadas? Interagir:pensando a extensão. 2009; 14:17-20.

8. Rodrigues VP, Lopes FF, Abreu TQ, Neves MIR, Cardoso NC. Avaliação dos hábitos de higiene bucal de crianças durante o período de internação hospitalar. Odontol Clín Cient Recife. 2011; 10(1):49-55

9. Cruz MCFN, Valois EM, Libério SA, Lopes FF. Avaliação clínica das alterações de mucosa bucal em crianças hospitalizadas de 3 a 12 anos. RGO. 2008; 56(2):157-61.

10. Gaetti-Jardim E, Setti JS, Cheade MFM, Mendonça JCG. Atenção odontológica a pacientes hospitalizados: revisão da literatura e proposta de protocolo de higiene oral. R Bras Ci Saúde. 2013;11(35):31-6.

11. Mattevi GS, Figueiredo DR, Patrício ZM, Rath IBS. A participação do cirurgião dentista em equipe de saúde multidisciplinar na atenção à saúde da criança no contexto hospitalar. Ciênc saúde coletiva. 2011; 16(10):4229-36.

12. Costa DC, Saldanha KFD, Sousa AS, Gaetti-Jardim EC Perfil de saúde bucal dos pacientes internados no Hospital Universitário Maria Aparecida Pedrossian, Campo Grande (MS). Arch Health Invest. 2016; 5(2):70-7.

13. Aguiar ASW, Guimarães MV, Morais RMP, Saraiva JLA. Atenção em saúde bucal em nível hospitalar: relato de experiência de integração ensino/serviço em odontologia. Extensio. 2010; 7(9):100-10.

14. Almeida TF, Torres AS, Silva RA, Wanderley FGC, Fonseca E. Avaliação dos cuidados de saúde bucal em pacientes pediátricos hospitalizados. Rev Ciênc Méd Biol. 2014; 13(1):72-7.

15. Miranda AF. The Dental surgeons in the hospital and their professional areas in Brazil: hospitalization units, surgical centers and intensive care units. J Community Med Health Educ. 2017; 7(1):505 doi:10.4172/21610711.1000505

16. Morais TMN, Silva A, Avi ALRO, Souza PHR, Knobel E, Camargo LFA. A importância da atuação odontológica em pacientes internados em unidade de terapia intensiva. Rev Bras Ter Intensiva. 2006; 18(4):412-7.

17. Barbosa AM, Ribeiro DM, Caldo-Teixeira AS. Conhecimentos e práticas em saúde bucal com crianças 
hospitalizadas com câncer. Ciênc saúde coletiva. 2010; 15(suppl 1):1113-22.

18. Oliveira LS, Bernardino IM, Silva JAL, Lucas RSCC, d'Avila S. Conhecimento e prática do controle de higiene bucal em pacientes internados em unidades de terapia intensiva. Rev ABENO. 2015;15(4):29-36.

19. Chapper A, Goldani MZ. A participação de odontólogos em equipes multidisciplinares. Rev Fac Odonto, Porto Alegre. 2004; 45(2):3-5.

20. Brasil. Ministério da Saúde. Secretaria de Atenção à Saúde. Secretaria de Vigilância em Saúde. SB Brasil 2010: Pesquisa Nacional de Saúde Bucal: resultados principais. Brasília: Ministério da Saúde, 2012.

21. Moraes Grisi MF, Corrêa Grisi D, Souza SLS, Macedo GO. Exame clínico em Periodontia. Periodontia: a atuação clínica baseada em evidências científicas, São Paulo: Artes Médicas; 2005.

22. Larson R, Farber B. Estatística Aplicada. 6. ed. São Paulo: Pearson Prentice Hall; 2016.

23. O'Donnell O. Access to health care in developing countries: breaking down demand side barriers. Cad Saude Pública. 2007; 23(12):2820-34.

24. Jaber KY, Franzi AS, Sassi LM, Rapoport A, Guebur MI, Dedivitis RA. Triclosan versus clorexidina no controle químico da placa e da gengivite em pacientes dentados com carcinoma espinocelular de boca, submetidos à radioterapia pós-operatória. Rev Bras Cir Cabeça e Pescoço. 2007; 36(2):75-9.

25. Miñana V. Grupo Previnfad/papps infancia y adolescência: Promocion de la salud buco dental. Rev Pediatr Aten Primaria. 2011; 13(51):435-58.

26. Ximenes RCC, Aragão FSD, Colares V. Avaliação dos cuidados com a saúde oral de crianças hospitalizadas. Rev Fac Odontol, Porto Alegre. 2008; 49(1): 21-5.

27. Motta WKS, Nóbrega DRM, Santos MGC, Gomes DQC, Godoy GP, Pereira JV. Aspectos demográficos e manifestações clínicas bucais de pacientes soropositivos para o HIV/Aids. Rev Odontol UNESP. 2014; 43(1):61-7.

28. Jahanbani J, Sandvik L, LybergT, Ahlfors E. Evaluation of oral mucosal lesions in 598 referred Iranian patients. Open Dental J. 2009; 3:42-7.

29. Riobbo-Crespo Mdel R, Planells-del Pozo P, RiobboGarcía R. Epidemiología de la patología de la mucosa oral más frecuenteen niños Epidemiology of the most common oral mucosal diseases in children. Med Oral Patol Oral Cir Bucal. 2005; 10(5):376-87.

30. Yilmaz AE, Gorpelioglu C, Sarifakioglu E., Dogan DG, Bilici M, Celik N. Prevalence of oral mucosal lesions from birth to two years. Niger J Clin Pract. 2011; 14(3):349-53

31. Majorana A, Bardellini E, Flocchini P, Amadori F, Conti $\mathrm{G}$, Campus G. Oral mucosal lesions in children from 0 to 12 years old: ten years' experience. Oral Surg Oral Med Oral Pathol. 2010; 110(1):13-8.

32. Bessa CFN, Santos PJB, Aguiar MCF, Do Carmo MAV. Prevalence of oral mucosal alterations in children from 0 to 12 years old. J Oral Pathol Med. 2004; 33(1):17-22.

33. Crivelli MR, Muhlmann M, Adler I, Cornicelli JC. Prevalecía de patología bucal em niños. Rev Asoc Odontol Argent. 1986; 74(3):81-2.

34. Santos PSS, Mariano M, Kallas MS, Nunes MC. Impact of tongue biofilm removal on mechanically ventilated patients. Rev Bras Ter Intensiva 2013; 25(1):44-8.
35. Panagakos FS, Scannapieco F. Periodontal Inflammation: from gingivitis $\mathrm{t}$ systemic disease. In Tech: Croácia; 2011. p. 230.

36. Iquejiri $\mathrm{MH}$, Zárate-Pereira P. Influência dos aspectos socioeconômicos na incidência da gengivite. Rev. Int. Periodontia Clin. 2005; 2(6/7):107-14.

37. Antunes JLF, Peres MA, Mello TRC. Determinantes individuais e contextuais da necessidade de tratamento odontológico na dentição decídua no Brasil. Rev C S Col. 2006; 11(1):79-87.

38. Freitas-Aznar AR, Capelozza ALA, Aznar FDC, Soares Junior LAV, Santos PSS. A bioética no contexto da Odontologia Hospitalar: uma revisão crítica. Rev bras odontol. Rio de Janeiro. 2016; 73(4):311-4.

\section{CONFLITO DE INTERESSES}

Os autores declaram não haver conflitos de interesse.

\section{AUTOR PARA CORRESPONDÊNCIA}

Niebla Bezerra de Melo

niebla.melo@gmail.com

Submetido em 26/03/2017 Aceito em 02/05/2017 